\title{
Characteristics and forecasting of respiratory viruses epidemic among children in west China
}

\author{
Wensheng $\mathrm{Li}^{1}$, Jinlan $\mathrm{He}^{2}$, Yali Cui ${ }^{1}$, Chunyu $\mathrm{Gan}^{1}$, Yu Gou${ }^{1}$, and Yifei Duan ${ }^{1}$ \\ ${ }^{1}$ Sichuan University West China Second University Hospital \\ ${ }^{2}$ Sichuan University West China Hospital
}

August 4, 2020

\begin{abstract}
Objectives: To assess the characteristics and forecasting of respiratory viruses epidemic among young children with acute respiratory tract infection (ARTI) in west China. Methods: This study retrospected the epidemic characteristics of respiratory viruses among 11813 pediatric patients with ARTI between March 2018 and March 2020. Results: 11813 samples were collected, with a mean age of 2.25 years. The ratio of the boy: the girl was 1.36 . The two predominant viruses were influenza (Flu) A and respiratory syncytial virus (RSV), with Flu A accounting for $47.3 \%$ ( $\mathrm{n}=1099), 47.5 \%$ ( $\mathrm{n}=1333$ ) and RSV accounting for $32.7 \%(\mathrm{n}=760), 24.7 \%(\mathrm{n}=692)$ of the positive samples in the two respective years. The positive rate of Flu B was $10.9 \%$ and $13.1 \%$, and the other four viruses were $<7 \%$. The most common virus was RSV in the $<5$ years group and Flu A in the 5-10 years group. Flu A and RSV demonstrated pronounced seasonality, and their infection rates increased from October. During the pandemic of SARS-CoV-2, isolation measures led to a decline in the number of ARTI. Conclusions: The study provided surveillance of respiratory viruses in west China. It will provide guidance for medical staff to implement the necessary prevention and management strategies before the outbreak of the virus in the future.
\end{abstract}

\section{Introduction}

ARTI is the leading cause of morbidity and mortality in young children. ${ }^{1}$ In 2015,138 million ARTI cases, 22 million severe ARTI cases and 0.9 million ARTI deaths occurred globally. The majority of ARTI can be attributed to viral infection, and among diverse respiratory viruses, respiratory syncytial virus (RSV), ADV, influenza (Flu) and parainfluenza virus (PIV) are the most common ones. ${ }^{2-5}$ According to WHO statistics, RSV infection causes 3.2 million people to be hospitalized, and $1 / 15$ of them eventually die. ${ }^{6}$ Flu, with an obvious seasonality in positive infection rate, accounts for $7 \%$ of ARTI cases, $5 \%$ of ARTI hospital admissions, and $4 \%$ of ARTI deaths in children $<5$ years. ${ }^{7}$ ADV infection can be seen all the year round, accounting for $5 \%$ to $10 \%$ of lower respiratory tract infections in children. ${ }^{8}$

After the outbreak of SARS in 2003, China Information System for Disease Control and Prevention (CISDCP), the notifiable disease reporting system, was overhauled. However, the sudden emergence of SARSCoV-2, highlighted new threats and need for more effective surveillance. ${ }^{9}$ The real time broadcast of virulent viruses such as SARS-CoV-2 during the outbreak raised the awareness that the epidemic of common clinical respiratory viruses such as RSV, ADV and Flu should also be monitored and reported because they also cause great health burden. Moreover, the broadcast of many infectious diseases often covered the overall situation of the whole country, lacking epidemic situation in specific provinces or cities. In addition, the data obtained were often confirmed positive severe cases reported by local medical institutions, but many mild outpatient cases have not been included, resulting in the loss of some positive data. Therefore, it's quite important to review and update the epidemiological characteristics of respiratory viruses in our region.

A comprehensive and up-to-date epidemiological data on viral ARTI would be vital for clinical management 
especially in warning the possible time of the next outbreak of the respiratory viruses. ${ }^{10}$ Furthermore, it provides clinicians with priority for virus detection in children, because early identification of virus can reduce the treatment time by 2.5 to 4 days. ${ }^{11}$ Therefore, we conducted a retrospective study on the detection results of respiratory viruses in children with ARTI, aiming to determine the etiologies of viral ARTI in west China. Through the analysis of the prevalence of the seven respiratory viruses in the past two years, we want to find out the susceptible population of respiratory viruses, the common types of viruses, the epidemic season and the changes of the viral positive rate before and during the outbreak of SARS-CoV-2.

\section{Methods}

\section{Study population}

This study was conducted between March 3, 2018 and March 3, 2020, as no positive sample of any virus was detected from March 4 to June 3, 2020. Children aged 0-10 years old with ARTI who came to see pediatricians in our hospital had their nasopharyngeal swabs collected and sent for analysis $(n=11813)$. Because the specimens were collected at the time of admission or outpatient service, we could fully exclude the origin of ARTI in hospital. Upon receiving the nasopharyngeal swabs, the laboratory technicians would process and test the samples immediately.

\section{Specimen detection}

All of the seven most common respiratory viruses were tested in each child, among which PIV l, PIV 2, PIV 3, RSV and ADV were detected by direct immunofluorescence assay using the $\mathrm{D}^{3}$ Ultra DFA Respiratory Virus Screening and ID Kit (Diagnostic Hybrids, Inc., USA), and Flu A and F1u B were detected by colloidal gold method using the Influenza A\&B Antigen detection Kit (Wondfo Biotech Co., Ltd, China). All the batch numbers of the reagents are within the period of validity, and the standard reference substance of the reagent was taken with each test.

\section{Viral identification}

For the identification of PIV l, PIV 2, PIV 3, RSV and ADV, samples were observed under Olympus BX43 LED fluorescence microscope after direct immunofluorescence staining. Samples were considered qualified only when the number of epithelial cells was more than 20, which was judged by at least 2 epithelial cells in each random field at $200 \times$ magnification. When more than 2 green fluorescent cells were found in visual field, the samples were judged to be positive. When samples were tested for Flu A and F1u B by colloidal gold method, the quality control line should appear regardless of whether a test line appears, and the result was judged to be positive if the test line appeared.

\section{Statistical analysis}

Data were analyzed with SPSS Statistics 19 (SPSS, Inc., USA). Distribution normality was checked for all variables including age and gender. The comparison of the age was analyzed by Mann-Whitney U test. Due to the unequal group sizes and the data variance, Chi-square test or Fisher's exact test was used to analyze the association between age, gender and year groups with respiratory viruses. A P value of $<0.05$ was considered statistically significant.

\section{Results}

A total of 11813 samples were collected in two years, and the socio-demographic variables of all the samples were outlined in Table 1. In the first year (March 3rd, 2018 to March 3nd, 2019), 82.8\% (4294/5184) patients were less than 5 years old. The mean age of the children was $2.2(2.5)$ years old, with more than half $(58.1 \%)$ of the patients being less than 1 year old. In the second year (March 4th, 2019 to March 3rd, 2020), 81.7\% $(5414 / 6629)$ of patients were less than 5 years. The mean age of the children was 2.3 (2.5) years old, with $45.4 \%$ of the patients being less than 1 year old. There was no significant difference either in the mean age $(\mathrm{P}=0.085)$ or in the constituent ratio of age $(\mathrm{P}=0.102)$ between the two years. The total number of samples from boys were higher than that from girls $(\mathrm{P}<0.01)$. The ratio of boy: girl was 1.36 and 1.37 in two respective years, without statistical difference $(\mathrm{P}=0.85)$. 
The overall positive detection rates of the all the samples were $44.8 \%$ in the first year and $42.3 \%$ in the second year, without significant difference $(\mathrm{P}=0.09)$. The two predominant viruses were Flu A and RSV in both years, with Flu A accounting for $47.3 \%, 47.5 \%$ and RSV accounting for $32.7 \%, 24.7 \%$ of the positive samples in the two respective years. The third predominant virus was Flu B and the positive rates of the other four viruses were low. Though there was no significant difference in the overall positive rates between the two years, especially the positive rates of Flu A $(\mathrm{P}=0.93)$, the positive rate of RSV in the first year was significantly higher than that in the second year $(\mathrm{P}<0.001)$.

$35.1 \%$ of children with positive viruses were below 5 years old in the first year. In this age group, Flu A and RSV were significantly more common $(\mathrm{P}<0.01)$. The proportion of RSV decreased to $1.6 \%(\mathrm{P}<0.01)$ while that of the Flu A increased significantly to $77.2 \%(\mathrm{P}<0.01)$ in the $5-10$ years group. In the second year, $31.9 \%$ of children with positive viruses were below 5 years old. Flu A and RSV, accounting for $40.7 \%$ and $31.7 \%$ of the total positive viruses, were significantly more common $(\mathrm{P}<0.01)$. However, Flu $\mathrm{B}$ was more common than RSV ( $20.7 \%$ vs. $10.6 \%, \mathrm{P}<0.01)$ in the $5-10$ years old age groups. In both years, the virus positive detection rates of children in $<5$ years group $(>30 \%)$ was significantly higher than those in the high age group (around 10\%).

Among the children who came to our hospital because of ARTI, the number of boys was significantly higher than that of girls. From all the positive samples, $25.8 \%$ were boys and $19.0 \%$ were girls in the first year, $25.0 \%$ were boys and $17.3 \%$ were girls in the second year. The constituent ratio of boy: girl has no statistical significance between the two years $(\mathrm{P}=0.34)$. The positive detection rates in boys were of no significant difference with those in girls $(44.9 \%$ vs. $44.7 \%, \mathrm{P}=0.96$ and $43.2 \%$ vs. $41.1 \%, \mathrm{P}=0.27$ in two respective years $)$, and differences between gender were not statistically significant for the seven viruses $(\mathrm{P}>0.05)$.

The overall distribution of samples in both years was shown in Figure 1. The seasonal distributions of each respiratory virus in the first and second year were shown in Figure 2 and Figure 3, respectively. Flu A and RSV demonstrated pronounced seasonality, with peak infection occurred in autumn and winter (from October to December), and lowest activity in early of the year (from April to August). Flu B had a small peak in January and March, but the number of positive cases in other months was small. Because of the higher total number of children in the second year, the monthly positive number in the second year was always higher than that in the first year except in January. There were 586 positive samples in January 2019, while in comparison the positive number in January 2020 decreased significantly to 354 . In order to eliminate the influence of the difference in the total number of children tested, we converted the number of infections to the positive rate of each month. The number of other viruses was too small to detect any seasonality, so we only made analysis on the positive rates of Flu A and RSV. As shown in Figure 4, the monthly positive rates of Flu A and RSV were consistent in two years with obvious seasonality.

\section{Discussion}

Many kinds of viruses can cause ARTI with various clinical symptoms. Early detection of virus is not only beneficial for the recovery from infection, ${ }^{12}$ but also conducive to the control of virus outbreaks so as to avoid wider spread. Besides, it also strongly helps epidemiological investigation. ${ }^{13}$

The present study is the first one in the past decade to investigate the epidemiology of respiratory viruses in west China. We found that the total number of children with ARTI in the second year was more than that in the first year. In both years, there were more boys than girls with similar boy: girl ratios. The majority of children with ARTI were young boys, indicating that boys were more vulnerable to ARTI than girls. ${ }^{14}$ The average age of the population was only $2.2 \sim 2.3$ years old, and nearly $50 \%$ of the children were less than 1 year old. The heavier burden of viral respiratory infections in children less than 1 year might be a result of an immature immune system and waning of maternal antibodies after 6 months old. Then, children are able to develop antibodies by themselves between the age of 2.5 to 3.5 years old to prevent viral infections. ${ }^{15}$

Previous epidemiological data showed that RSV was the most predominant respiratory virus in children. ${ }^{16}$ However, our data revealed that Flu A was the most common virus in our region. It could be caused by the usage of more sensitive detection methods or the seasonal variations nature of virus distributions. Our 
results are aligned with a study in which Flu A was found to be the most predominant virus among Chinese young children, followed by RSV. ${ }^{17}$ In our study, RSV was predominantly detected in children less than 5 years old, while Flu A and B were mainly in 5 10 years old, suggesting that RSV is likely to be infected in the family or community, while Flu A and B might mainly be infected in school.

The characteristics of geographical areas could significantly affect the observed burden of each respiratory virus. ${ }^{9}$ The weather in west China belongs to subtropical monsoon humid climate. The monthly positive rates of the two years were mostly similar, indicating the positive rate of viruses remains almost unchanged in the same region unless there were a large number of imported cases. The monthly trend has been consistent until January. The highest burden of viral infection in the first year was recorded in January, while in comparison the positive number in January 2020 decreased significantly, mainly due to measures against SARS-CoV-2 such as strict home isolation and long-term wearing of masks, which could effectively block the spread of the viruses. The sharp decrease in the number of infection was consistent with the data provided by the China National Influenza Center on 5 June $2020^{18}$, proving that measures taken by our country against SARS-CoV2 were completely effective. ${ }^{19}$ Compared with other viruses, the positive rates of Flu A and RSV were higher throughout the study period. We knew that Flu A and RSV have obvious seasonality. The positive number increased when the weather got colder (autumn and winter) and decreased when the weather was warmer (spring and summer). The total number of positive samples increased from October in the past two years. Therefore, it's reasonable to infer that the infection rate of respiratory virus in 2020 may gradually increase from October if less strict isolation measures would be taken because of the blockage of SARS-CoV-2 in China.

Due to the timely adoption of various isolation measures, SARS-CoV-2, which broke out in late 2019, did not break out widely in our region. Besides, few cases have been observed in children and adolescents because they have a more favorable clinical course than adult. ${ }^{20}$ From the outbreak to 3 June 2020, our hospital has tested all presumed cases for SARS-CoV-2 nucleic acid, as well as SARS-CoV-2 antibody tests for 21241 hospitalized patients and their families, but none of the test results were positive. Therefore, the possible influence of SARS-CoV-2 on the positive rate of the seven respiratory viruses in children could be excluded.

\section{Conclusion}

In summary, this study provided important virus surveillance of ARTI burden and seasonality among young children in west China. The variety of respiratory viruses detected in this study highlighted the need for a monitoring database of ARTI and its etiological pathogen among children of different ages and genders. Judging from the epidemic season of respiratory virus in our region, we infer that the infection rate of respiratory virus in 2020 may gradually increase from October, and people can take corresponding preventive measures in advance to reduce the risk of viral infection.

\section{References}

1. Wang X, Li Y, O'Brien KL, et al. Global burden of respiratory infections associated with seasonal influenza in children under 5 years in 2018: a systematic review and modelling study. The Lancet Global health. 2020;8(4):e497-e510.

2. Luo HJ, Huang XB, Zhong HL, et al. Epidemiological characteristics and phylogenic analysis of human respiratory syncytial virus in patients with respiratory infections during 2011-2016 in southern China. Int $J$ Infect Dis.2020;90:5-17.

3. Kong DC, Zheng YX, Jiang CY, et al. [Analysis of adenovirus infection in acute respiratory tract infection cases in Shanghai from 2015 to 2019]. Zhonghua Liu Xing Bing Xue Za Zhi. 2020;41(5):733-737.

4. Ma Y, Liu K, Yin Y, et al. The Phylodynamics of Seasonal Influenza A/H1N1pdm Virus in China Between 2009 and 2019. Front Microbiol. 2020;11:735.

5. Wen S, Yu M, Zheng G, et al. Changes in the etiology of viral lower respiratory tract infections in hospitalized children in Wenzhou, China: 2008-2017. 2019. 
6. Pangesti KNA, El Ghany MA, Kesson AM, Hill-Cawthorne GA. Respiratory syncytial virus in the Western Pacific Region: a systematic review and meta-analysis. J Glob Health. 2019;9(2):020431.

7. Lafond KE, Nair H, Rasooly MH, et al. Global Role and Burden of Influenza in Pediatric Respiratory Hospitalizations, 1982-2012: A Systematic Analysis. PLoS Med.2016;13(3):e1001977.

8. Jin Y, Zhang RF, Xie ZP, et al. Prevalence of adenovirus in children with acute respiratory tract infection in Lanzhou, China. Virol J. 2013;10:271.

9. Dong Y, Wang L, Burgner DP, et al. Infectious diseases in children and adolescents in China: analysis of national surveillance data from 2008 to 2017. 2020;369:m1043.

10. Hui DS, Ng SS. Recommended hospital preparations for future cases and outbreaks of novel influenza viruses. Expert Rev Respir Med. 2020;14(1):41-50.

11. Moesker FM, van Kampen JJA, Aron G, et al. Diagnostic performance of influenza viruses and RSV rapid antigen detection tests in children in tertiary care. Journal of clinical virology : the official publication of the Pan American Society for Clinical Virology. 2016;79:12-17.

12. Kolawole O, Oguntoye M, Dam T, Chunara R. Etiology of respiratory tract infections in the community and clinic in Ilorin, Nigeria. 2017;10(1):712.

13. Giamberardin HI, Homsani S, Bricks LF, et al. Clinical and epidemiological features of respiratory virus infections in preschool children over two consecutive influenza seasons in southern Brazil. J Med Virol. 2016;88(8):1325-1333.

14. Yassine HM, Sohail MU, Younes N, Nasrallah GK. Systematic Review of the Respiratory Syncytial Virus (RSV) Prevalence, Genotype Distribution, and Seasonality in Children from the Middle East and North Africa (MENA) Region. Microorganisms.2020;8(5).

15. Yew SM, Tan KL, Yeo SK, Ng KP, Kuan CS. Molecular epidemiology of respiratory viruses among Malaysian Young children with a confirmed respiratory infection during 2014-2015. Journal of thoracic disease. 2019;11(11):4626-4633.

16. Lu L, Robertson G, Ashworth J, et al. Epidemiology and Phylogenetic Analysis of Viral Respiratory Infections in Vietnam. Front Microbiol. 2020;11:833.

17. Li Y, Wang J, Wang C, et al. Characteristics of respiratory virus infection during the outbreak of 2019 novel coronavirus in Beijing. Int $J$ Infect Dis.2020;96:266-269.

18. Weekly Influenza Report. 2020; http://ivdc.chinacdc.cn/cnic/zyzx/lgzb/202006/t20200605_217160.htm . Accessed 5/6, 2020.

19. Dong Y, Mo X, Hu Y, et al. Epidemiology of COVID-19 Among Children in China. Pediatrics.2020;145(6).

20. De Rose DU, Piersigilli F, Ronchetti MP, et al. Novel Coronavirus disease (COVID-19) in newborns and infants: what we know so far. 2020;46(1):56.

Table 1. Distribution of respiratory viruses according to age and gender in different years

Variables

Table 1. Distril 2018.3.3〜2019.3.

Samples (\% of total sample received)

Total sample received

Age n (\%)

$<5$ years

$(100.0)$

(100.0)

Samples (\% of to 4294 (82.8)

5414 (81.7)

Positive samples

$1822(35.1)$

(44.8)

2116 (31.9) 
(42.3)

Respiratory virus identified (\% of total positive samples within age/gender)

Influenza A

$(47.3)$

$(47.5)$

Influenza B

$(10.9)$

(13.1)

Parainfluenza virus 1

$(0.8)$

(1.9)

Parainfluenza virus 2

(0.7)

Parainfluenza virus 3

$(4.8)$

(6.8)

Respiratory syncytial virus

(32.7)

(24.7)

Adenovirus

(3.5)

(5.4)
Respiratory virus

712 (39.1)

$861(40.7)$

$170(9.3)$

224 (10.6)

$15(0.8)$

$48(2.3)$

0

107 (5.9)

$184(8.7)$

$752(41.3)$

671 (31.7)

66 (3.6)

113 (5.3)

Figure 1

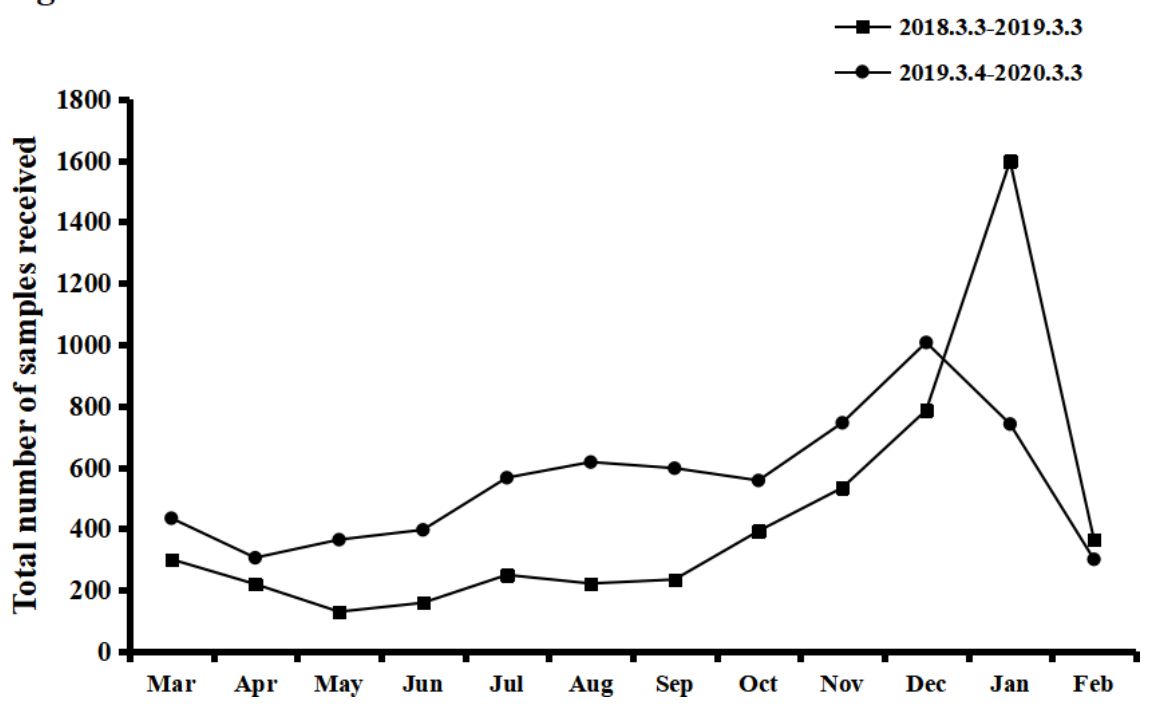


Figure 2

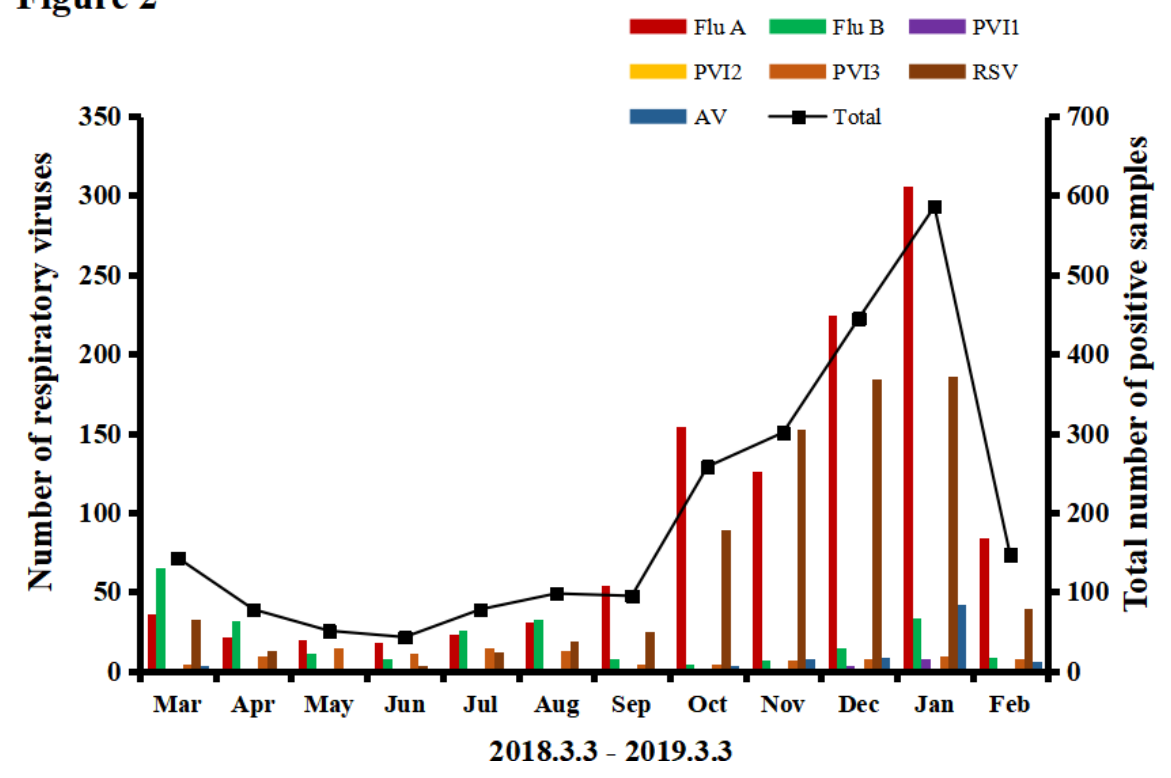

Figure 3

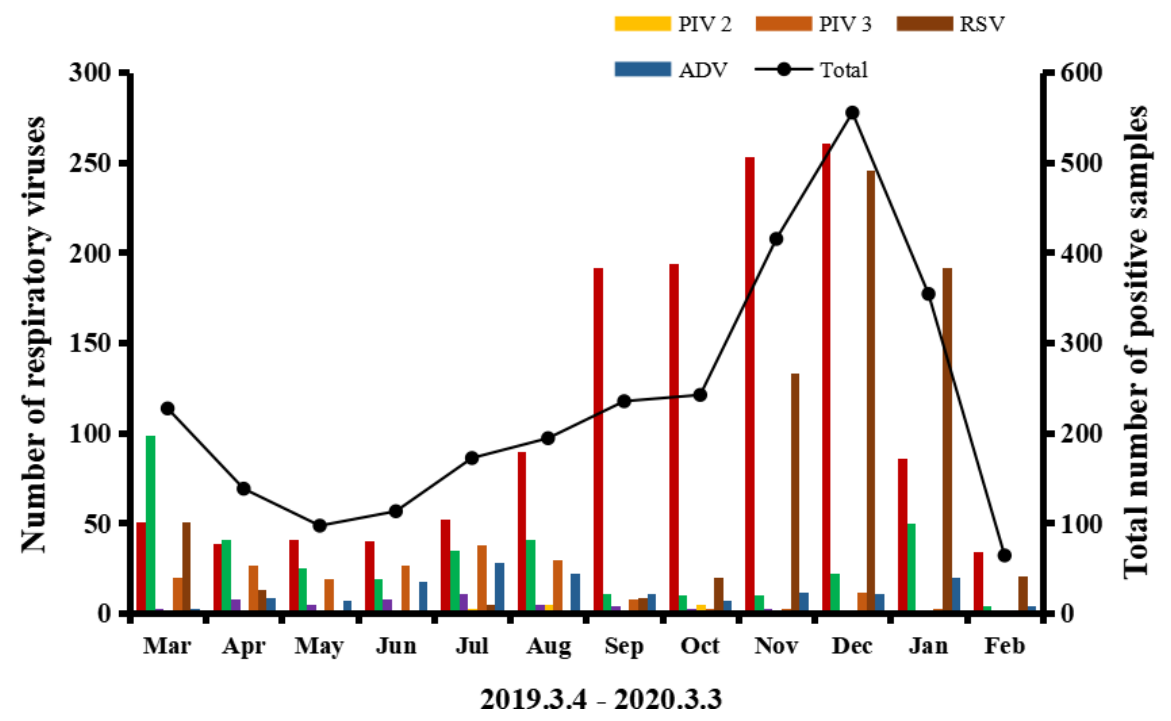


Figure 4

—2018-2019 Flu A 明明明 2019-2020 Flu A

2018-2019 RSV 明明明 2019-2020 RSV

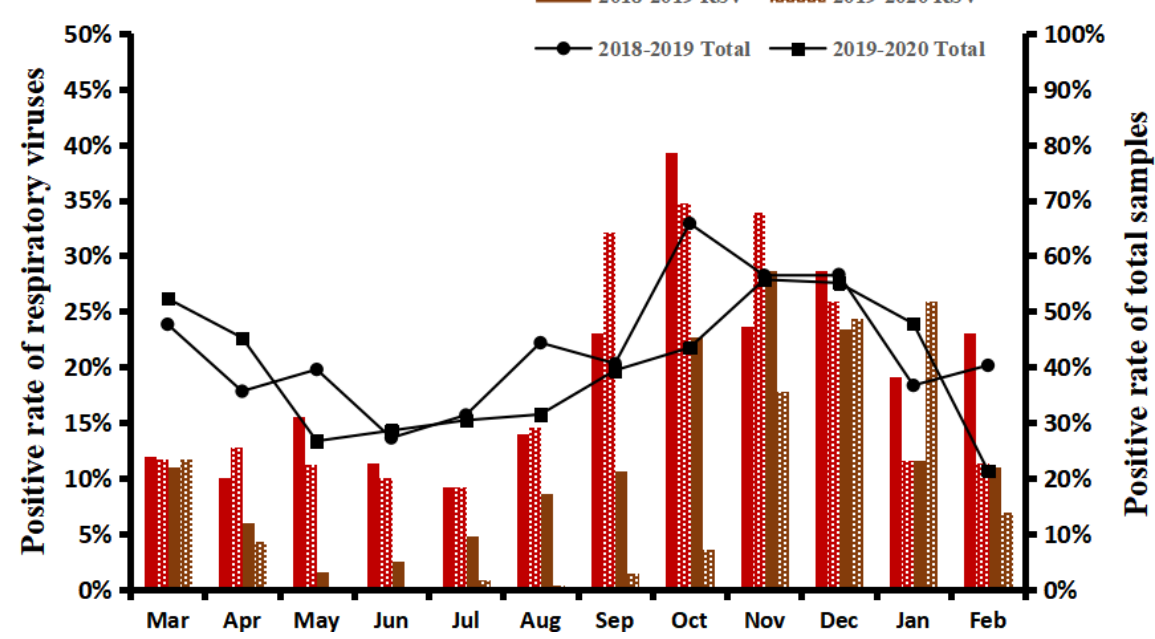

